\title{
¿Organización cultural o asociación ilícita? Reyes y reinas latinos entre Madrid y Barcelona
}

\author{
Carles Feixa $^{1}$ \\ Bárbara Scandroglio ${ }^{2}$ \\ Jorge S. López Martínez 3 \\ Francisco Ferrándiz ${ }^{4}$ \\ 1. Universitat de Lleida \\ feixa@geosoc.udl.cat \\ 2. Universidad Autónoma de Madrid \\ barbara.scandroglio@uam.es \\ 3. Universidad Autónoma de Madrid \\ jorge.lopez@uam.es \\ 4. ILLA-CCHS-CSIC. Madrid \\ fferrandiz@ile.csic.es
}

Recibido: 30-03-2008

Aceptado: 10-11-2008

\section{Resumen}

El artículo se propone mostrar que el diverso tratamiento social otorgado a la presencia de «reyes» $\mathrm{y}$ «reinas» latinos en diferentes lugares de España ha justificado distintos planteamientos de intervención defendidos fundamentalmente por los poderes públicos. Para ello, presentamos los primeros resultados de un proyecto de $\mathrm{I}+\mathrm{D}+\mathrm{i}$ en el que se ha realizado trabajo de campo en Barcelona y Madrid. Dichos contextos locales ejemplifican distintas modalidades de actuación. Para su comparación, nos centramos en dos acontecimientos que tuvieron gran repercusión en la opinión pública: la constitución de la Organización Cultural de Reyes y Reinas Latinos de Catalunya (julio de 2006) y el proceso por asociación ilícita contra el grupo de reyes y reinas latinos vinculados a la Sagrada Tribu América Spain en Madrid (abril de 2007). Dicha comparación revela que, más que a los rasgos socioculturales de los jóvenes migrantes, las diferencias responden a discrepancias en las políticas de acogida.

Palabras clave: cultura juvenil; jóvenes migrantes; Latin Kings \& Queens; violencia; transnacionalismo.

Abstract. Cultural organization or illicit association? Latin Kings between Madrid and Barcelona

This paper analyzes the ways in which the differential social attitude towards latin kings and queens in different parts of Spain results in substantially different public policies towards them. To prove this, we discuss the first results of an I-D+i public research project that has allowed us to carry out fieldwork with groups of Latin Kings \& Queens in Barcelona and Madrid. The policies in both cities are radically different, and so are the situations on the ground. This paper focuses in two moments of the process which had 
an important public impact: the investiture of the Organización Cultural de Reyes y Reinas Latinos in Catalonia (in July 2006), and the judicial process for illegal association against the group of Latin Kings linked to the Sagrada Tribu América Spain in Madrid (in April 2007). This comparison reveals that the differences in the situation of both collectives respond more to the nature and consequences of the public policies implemented than to some presumed sociocultural traits of the young migrants.

Key words: youth culture; migrant youth; Latin Kings \& Queens; violence; transnationalism.

\section{Sumario}

\section{Introducción}

2. Marco histórico, teórico y metodológico

3. Reyes y reinas latinos en Madrid

4. Reyes y reinas latinos en Barcelona
5. Discusión: ¿ Organización cultural o asociación ilícita?

Postscriptum (1 de diciembre de 2010)

Referencias bibliográficas

\section{Introducción}

Como investigadores dedicados al estudio de los fenómenos juveniles - $-\mathrm{y}$, para algunos de nosotros, de sus manifestaciones violentas-, no nos sorprende haber tenido que orientar en estos últimos años nuestro trabajo a la agrupación de los reyes y reinas latinos. A unos, por la novedad que supuso su aparición en España - y su idiosincrasia respecto de otras agrupaciones- $y$, a otros, porque su visibilización a partir de las manifestaciones violentas ha vuelto a oscurecer la problemática más general de la violencia juvenil, que no es ni novedosa ni desconocida en España. Es aquí donde nuestros intereses confluyen y conforman el objetivo que planteamos en este artículo, esto es, analizar el proceso que lleva del tratamiento social del fenómeno a la implementación de acciones para su control, poniendo en evidencia qué estrategias se convierten en argumentaciones justificativas de determinadas acciones. Pretendemos, así, poner a prueba la hipótesis de que el tratamiento social del fenómeno - fundamentalmente mediático, político y policial - justifica los principios que orientarán las acciones interventivas y preventivas, al margen de su eficacia a corto y largo plazo y, sobre todo, al margen de las manifestaciones conflictivas protagonizadas por una minoría de los miembros de la agrupación o su incidencia «real». Es más, las críticas dirigidas a la eficacia de las intervenciones que se pretenden implementar, el dimensionamiento de la incidencia de las conductas de riesgo y, también, el manejo de las fuentes son parte estratégica del proceso de justificación analizado. Para alcanzar el objetivo que nos hemos propuesto, hemos analizado los primeros resultados de un proyecto de $\mathrm{I}+\mathrm{D}+\mathrm{i}^{1}$ en el que se ha

1. ¿Reyes y reinas latinos? Identidades culturales de los jóvenes de origen latinoamericano en España (SEJ2005-09333-C02-02/SOCI). 
realizado un trabajo de campo con grupos de reyes y reinas latinos de Barcelona y Madrid. Dichos contextos servirán para ejemplificar cada uno de los dos planteamientos considerados, centrándonos, para su comparación, en dos hechos: la constitución de la Organización Cultural de Reyes y Reinas Latinos de Catalunya (julio de 2006), por un lado, y el proceso por asociación ilícita contra el grupo de reyes y reinas latinos vinculados a la Sagrada Tribu América Spain en Madrid (abril de 2007), por el otro, así como en los efectos posteriores de uno y otro suceso.

\section{Marco histórico, teórico y metodológico}

El temor a las bandas juveniles violentas ya es una realidad.

(La Vanguardia, 02/11/2003)

Surgidos en Chicago al final de la Segunda Guerra Mundial, cuando confluyen diversos gangs puertorriqueños, dominicanos, cubanos, etc., no es hasta fines de los años ochenta cuando los Latin Kings aparecen en la escena pública y se difunden por otras zonas del país. La constitución del "capítulo" de Nueva York, que tiene lugar en la prisión de Collins en 1986, resulta de particular importancia. Un joven preso de origen cubano (conocido bajo el seudónimo de King Blood) entra en la banda y se erige como el líder supremo. En 1996, se elige a un nuevo líder (King Tone), que empieza a dar a los Latin Kings una dirección más política, centrada en la vindicación de la identidad latina y la condena de la brutalidad policial (Kontos, 2003). La banda (en realidad una compleja confederación de grupos locales) es rebautizada con el nombre de Almighty Latin King Nation (Todopoderosa Nación de los Reyes Latinos), y después se añade a ella la versión femenina (Latin Queens). A partir de aquí, se empiezan a generar una serie de producciones culturales (manifiestos, revistas, murales, sitios web) que desembocan en la Biblia Latin King (compilación de textos generados por los propios líderes). Ello supone la conversión del modelo de la «banda» territorial y con una matriz masculina y desviante a otro más cercano a un «movimiento social» desterritorializado y con una matriz más plural en su composición de género y en sus finalidades sociales. Contamos con una sugerente investigación etnográfica realizada durante esta «fase reformista» sobre esta transición que dista de ser pacífica y unívoca y a la que siguen oponiéndose poderosas fuerzas internas y externas (Brotherton y Barrios, 2003). A la difusión nacional, le seguirá la expansión internacional (primero en América Latina y posteriormente en Europa), lo que acaba convirtiendo a los Latin Kings en una especie de "franquicia» transnacional con múltiples conexiones "glocales». Aunque los nodos locales se adscriban a este imaginario original, sus expresiones concretas son muy heterogéneas e integran, en esta original matriz norteamericana representada por el modelo de gang, otras matrices tales como la latinoamericana, representada por el modelo de las pandillas o naciones; la transnacional, representada por los estilos juveniles subculturales, conocidos en España con el nombre de «tribus urbanas», y la virtual, representada 
por modelos identitarios juveniles que circulan por Internet (Matza, 1973; Feixa et al., 2006). De aquí que, aunque las bandas juveniles están estrechamente vinculadas al proceso de urbanización de los Estados Unidos y al proceso de «recuperación mágica» de la identidad étnica original por parte de las segundas y terceras generaciones de jóvenes cuyos padres o abuelos fueron migrantes, lo que se tradujo en el modelo de la banda territorial, cohesionada y básicamente masculina estudiada por los clásicos de la etnografía urbana (Thrasher, 1926; Whyte, 1943), en las últimas décadas se ha experimentado una evolución hacia formas de sociabilidad más complejas y desterritorializadas (Klein et al., 2001; Hagedorn, 2001). La historia de los Latin Kings \& Queens - considerados una de las mayores bandas norteamericanas en la actualidades, en este sentido, emblemática. Las identidades culturales de los jóvenes que pertenecen a agrupaciones como la de los Latin Kings \& Queens surgen, por tanto, en un territorio fronterizo donde, además de la cultura hegemónica y las culturas parentales, confluyen varias tradiciones subculturales. Se trata de identidades híbridas que corresponden a las culturas juveniles de la era de la información, en la que se yuxtaponen tradiciones locales y globales, presenciales y virtuales.

Las identidades híbridas de agrupaciones como los Latin Kings \& Queens constituyen un fenómeno complejo que permite múltiples análisis y que, de por sí, propicia que dichos análisis puedan ser instrumentados a partir de la exigencia de justificar, dependiendo del contexto sociopolítico y económico, unas intervenciones en vez de otras y para, como ha ocurrido reiteradamente a lo largo de la historia, crear "diablos populares» o chivos expiatorios. En Estados Unidos, existe una larga experiencia en el estudio y en la intervención de bandas desde diferentes perspectivas. A pesar de que la tradición criminológica ha tendido a enfatizar los aspectos delictivos de estos grupos, en el mundo académico existe cierto consenso a la hora de considerar que una banda es mucho más que un grupo delictivo, y que la mayor parte de la delincuencia urbana no tiene que ver con estos grupos (Klein, 1995). La obra de Brotherton y Barrios (2003) en base a los Latin Kings de Nueva York parte de un trabajo etnográfico que intenta hacer visibles los elementos políticos y de resistencia de comunidades étnicas subordinadas, elementos muy claros en el caso de los Latin Kings. En cualquier caso, la consideración de los Latin Kings \& Queens como grupo criminal dista de ser unánime: la mantienen aquellos que han trabajado con fuentes policiales y/o con testimonios de convictos y de exmiembros; la refutan, aún sin caer en una visión angelical ni «buenista», los que han llevado a cabo un trabajo de campo más intenso.

El análisis que se discute a continuación se ha basado, en primer lugar, en entrevistas formales e informales a miembros de la agrupación de los reyes y reinas latinos - en este caso, fueron tanto individuales como grupales- y a informantes clave (investigadores, abogados, miembros de los diferentes cuerpos de las fuerzas de seguridad, periodistas, educadores); en segundo lugar, en la observación de actos celebrados por la agrupación y de procesos judiciales, y, en tercer lugar, en documentos (material producido por la agrupación, pro- 
ducciones de los medios de comunicación, ponencias, declaraciones públicas, informes, autos de instrucción y sentencias de procesos judiciales). El análisis sigue el desarrollo del proceso intentando evidenciar las influencias mutuas entre el contexto social y la propia organización, así como teniendo en cuenta algunos de los hechos clave que se han producido en ambos contextos.

\section{Reyes y reinas latinos en Madrid}

Estuve mucho tiempo antes de formar la Nación aquí, porque es una responsabilidad muy grande y difícil. $Y$ es que la gente de aquí tiene un pensamiento muy diferente al de la Nación.

(King Wolverine, Manifiesto ALKQN Sagrada Tribu América Spain, 2001)

Según el «manifiesto» recogido en la «literatura», la Sagrada Tribu América Spain (STAS) fue fundada en Madrid el 14 de febrero de 2000 por su «padrino", miembro de la Sagrada Tribu Atahualpa Ecuador (STAE). El manifiesto está redactado dos años después y en él se nombran ${ }^{2}$ a quienes integraron el grupo inicial. Éste se constituirá en el Reino Inca, correspondiente a la Comunidad de Madrid. En un segundo momento, se constituye el Reino Hispano en Barcelona, correspondiente a la Comunidad de Cataluña. Contrastando el manifiesto con la información recogida en las entrevistas, la fundación de la STAS no corresponde a un momento preciso, y menos a la fecha indicada, en el que los jóvenes citados en él se hayan reunido a tal efecto o la hayan acordado conjuntamente. De los que formaron parte del núcleo inicial, algunos entraron después de la fecha indicada; otros en el momento de la redacción del manifiesto ya estaban alejados, y, finalmente, otros que sí estuvieron en los inicios no son nombrados. Además, aquellos que estaban de acuerdo en constituir una «tribu» independiente de Ecuador decidieron el nombre de STAS a principios del 2001 y, probablemente, puede considerarse ésta una fecha de fundación más próxima a los acontecimientos. El padrino, según otros, no vino de Ecuador ni con la intención de fundar la Nación ni con la carta con el permiso de fundar un capítulo de la Nación en España, aparte de que tuviera o no problemas personales con los encargados en Ecuador. La carta con el permiso llegó posteriormente de Ecuador con otro rey que, al parecer, tenía en realidad el encargo de sustituir al padrino al frente del grupo que ya se había constituido. Finalmente, la carta fue cedida al padrino. Siempre según el manifiesto, el motivo de la constitución de la tribu fueron las situaciones de abuso, de mal trato y brutalidad policial contra los inmigrantes latinoamericanos. Estas circunstancias, a las que se podría añadir la indiferencia o el rechazo de instituciones u organizaciones y la violencia ejercida por grupos de

2. Dadas las circunstancias en las que se encuentran actualmente los jóvenes miembros de la Nación en Madrid, no es posible ni ofrecer todos los datos recogidos a través de entrevistas formales e informales y la observación participante, ni ser precisos con los que se ofrecen. 
jóvenes españoles, sí son corroboradas y reconocidas por los reyes y las reinas entrevistados. En denunciar dichas circunstancias y en considerar la creación de la Nación en Madrid, una forma de hacerle frente, en efecto, han sido más resueltos otros miembros antes que el propio fundador.

En este punto de la investigación, no podemos todavía precisar lo que fue sucediendo internamente en el grupo desde este principio hasta que adquirió visibilidad mediática y comenzara la intervención policial a partir de 2003. Considerando los hechos delictivos imputados a supuestos miembros de la tribu, los recorridos seguidos por otros y algunas de las problemáticas señaladas por los entrevistados, la condición de jóvenes e inmigrados, el contexto socioeconómico y la historia previa de la propia Nación condicionaron que, por un lado, un número exiguo de sus integrantes emprendiera una escalada hacia conductas delictivas progresivamente más graves y, por otro, otros miembros comenzaran a involucrarse en enfrentamientos violentos con otros grupos, fundamentalmente compuestos por jóvenes latinoamericanos. En este sentido, que en el manifiesto se diga que los miembros de la tribu tienen que aprender a "reconocer, someter y tumbar con todo rencor» a sus enemigos -Discípulos, Blood, Crip, Netas y Vatos Locos-, es más bien un reflejo de lo que estaba aconteciendo con la tribu sobre el terreno que la consigna que ha determinado su devenir. Sin embargo, sería miope atribuir exclusivamente la progresiva decantación conflictiva de la Nación a las instrucciones de su supuesto fundador en vez de a las complejas dinámicas sociales en las que se veían envueltos paulatinamente. Además, el tratamiento mediático y político del fenómeno en Madrid ha propiciado que se atribuyeran a los Latin Kings - y se sigan atribuyendo en la actualidad - hechos delictivos de los que no han sido protagonistas y, por tanto, que se amplificara la percepción de la frecuencia y la gravedad de los mismos, con respecto a los casos probados o bien a otros contextos geográficos como, por ejemplo, Barcelona. Este pánico moral respecto a los Latin Kings, convertidos en una masa indiferenciada de presuntos delincuentes, ha sido utilizado para justificar, a nivel político, que en Madrid la única intervención juzgada viable fuera la «mano dura». O, como explicitó la directora de la Agencia de la Comunidad de Madrid para la Reeducación y la Reinserción del Menor Infractor, Carmen Balfagón Lloreda, «muy dura». Esta posición es coherente con la nueva e implacable «sensatez penal» transnacional de corte neoliberal originada en EEUU en torno a la doctrina de la "tolerancia cero", manifestada mediante prácticas de control social basadas en la criminalización sistemática de la pobreza y la diferencia (Wacquant, 2000; Greene y Pranis, 2007).

En este contexto, la iniciativa de comenzar el proceso de estudio para la posible constitución de una asociación en Madrid, promovida en octubre de 2006 por el entonces Defensor del Menor, Pedro Núñez Morgades, fue rechazada sin mayor consideración por la Dirección General de Justicia de la Comunidad de Madrid, cuyas representantes no acudieron a la reunión convocada para estudiar conjuntamente el proceso desarrollado en Barcelona (véase Álvarez, 2006). Las razones alegadas hacían alusión a la comisión de delitos 
por parte de supuestos miembros de los Latin Kings y a la intención, por ello, de lograr el reconocimiento del grupo como «banda organizada», señalando ya, a modo de profecía autocumplida y de forma previa al desarrollo del proceso judicial, el resultado esperado y previsto para el mismo. La línea de trabajo de los agentes institucionales del ámbito regional en este tema alcanzó ya la homogeneidad con la incorporación, en noviembre de 2006, del nuevo Defensor del Menor, Arturo Canalda González, quien señaló, en declaraciones recogidas en mayo de 2007 y refiriéndose a los Latin Kings (véase Madrid Diario/Europa Press, 2007), que «su futuro es la desaparición y eliminación», con lo cual marcaba una estrategia de acción que, con independencia de otras posibles valoraciones, quedaba centrada en la aplicación de medidas punitivas, incluso como única forma de prevención.

Sin embargo, si atendemos, por una parte, a las sentencias, las imputaciones o los datos proporcionados por los diferentes cuerpos de seguridad — que, en algunos años, no coinciden ni entre sí ni con los ofrecidos por la Delegación del Gobierno, incluso en el número de víctimas mortales atribuidas a «bandas latinas» — y, por otra parte, a su eco en los medios de comunicación —un caso manifiesto de amplificación e hipervisibilización mediática-, queda patente que ha sido más bien la resonancia social lo que ha determinado la percepción del fenómeno en los diferentes contextos - $\mathrm{y}$, por tanto, las medidas adoptadas- y no la frecuencia o la gravedad de los hechos. Más si se comparan con los atribuidos a otros grupos juveniles, tales como los skinheads de derecha o de izquierda. Especialmente en la Comunidad de Madrid, aunque no de forma exclusiva, esta percepción distorsionada ha justificado la sensación de asistir a una especie de "caza de brujas» — mediática, policial y judicial— socialmente orquestada. La admisión, por parte de periódicos, de artículos que vulneran las normas más elementales de rigor periodístico y que se basan únicamente en fuentes policiales; el uso estratégico e intensificado de los medios por parte de dichas fuentes; la persecución policial no justificada por hechos delictivos previos o, en caso que los hubiese, las detenciones indiscriminadas; la admisión de demandas de juicio presentadas por parte de la fiscalía pese a lo anterior; etc., no pueden separarse de este contexto político. Aunque debemos señalar que tal estrategia no ha sido la única respuesta institucional, ni siquiera en Madrid, donde algunos profesionales conocedores del terreno han optado por medidas de carácter más preventivo que represivo.

Dados los antecedentes, el juicio celebrado en mayo de 2007 en Madrid por asociación ilícita, coacciones y amenazas a catorce presuntos miembros de la Nación representó un momento de inflexión al que se encomendó una respuesta contundente, no sólo a la acción de las «bandas latinas», sino también a las pretensiones de los que habían optado o querían optar por otras vías de intervención sobre el fenómeno. En efecto, no sólo son muchos los elementos - detallados a continuación-que dejan patente su pretendido carácter «ejemplarizante», sino que la desacreditación durante la celebración del juicio de algunas pruebas y testigos protegidos, la liberación de los jóvenes imputados no condenados por otros delitos y no imputados en otras causas tres días 
después de que el juicio estuviera visto para sentencia y la propia sentencia han creado un evidente malestar entre quienes lo habían promovido ${ }^{3}$. Entre los elementos a los que hemos aludido, queremos destacar, en primer lugar, que nueve de las personas imputadas exclusivamente por los cargos contemplados en el proceso - y pese a los elementos probatorios que justificarán la sentencia- permanecieron en prisión preventiva durante más de un año y en medidas de aislamiento. Uno de ellos ni tan siquiera resultaría luego imputado -no sólo condenado- por elemento probatorio alguno en el proceso y, además, en el momento de su detención era menor de edad, condición que no se constató hasta llegado el juicio. En segundo lugar, en la semana anterior al inicio del juicio, se produjo la «filtración» completa y simultánea del informe de la fiscalía a algunos de los periódicos nacionales de mayor difusión. En cuarto lugar, los intentos de proponer claves de lecturas alternativas a las de la fiscalía, tanto por parte de los letrados de la defensa como de Carles Feixa, que intervino en calidad de perito, fueron descartadas por atender a factores de carácter "sociológico», por un lado, y por partir de la experiencia de otros contextos locales, nacionales o internacionales, por el otro. Pese a la intención de convertir el proceso en un "macrojuicio» que adquiriera gran visibilidad social, la cobertura otorgada al mismo y su resonancia no lograron despertar el interés pretendido en la opinión pública. Los medios de comunicación tuvieron que habérselas con declaraciones, documentos y elementos probatorios que no se correspondían ni a la alarma social ni a las expectativas previas. Además, eran material poco «noticiable», por no ofrecer elementos novedosos respecto a lo conocido sobre otros grupos juveniles que derivaban hacia la dinámica del enfrentamiento. La sentencia se hizo pública el 21 de junio de 2007 y, atendiendo una vez más a las reacciones de la prensa, puede considerarse salomónica. Se absuelven a todos los imputados de las acusaciones de coacciones y amenazas y se condenan algunos de ellos por falta de lesiones a la pena de un mes y quince días de multa. Además de ello, o pese a ello, se considera probada la asociación ilícita por la que se condenan once imputados a una pena de entre tres años y un año y medio de prisión, y se acuerda la disolución de la Sagrada Tribu América Spain de la Todopoderosa Nación de los Reyes y Reinas Latinos «en lo que respecta a la sección o "reino" establecido en la Comunidad Autónoma de Madrid (Reino Inca)». Los jueces reconocen que no puede considerarse ilícita dicha asociación desde sus principios, ya que sus objetivos eran acordes tanto con la convivencia como con la defensa de los inmigrantes latinoamericanos, además de que no constan actos delictivos cometidos por la asociación antes de 2004. Sin embargo, la agrupación fue degenerando con el

3. El 25 de mayo de 2007, antes de su intervención en las jornadas «Maras y pandillas juveniles: dos mundos diferentes», organizadas por el CIDOB, el capitán de la Guardia Civil Miguel López Corral declaró recibir con sorpresa la decisión del juez de excarcelar, antes del fallo, once de los supuestos miembros de los Latin Kings imputados en el juicio por asociación ilícita celebrado en la Audiencia Provincial de Madrid del 5 al 10 de mayo, ya que el trabajo de la Guardia Civil iba precedido por una larga investigación. 
tiempo «hacia una línea de actuación en la que primaron los enfrentamientos callejeros y las acciones violentas dentro y fuera del grupo" y, por tanto, «al haberse acreditado que en la organización se realizaban actos agresivos concretos contra los miembros internos del propio grupo, y ajustes de cuentas contra la banda contraria como una actividad primordial de la organización Latin King, no cabe más que concluir que tenía como un objetivo fundamental la comisión de actos delictivos».

No queremos ni ignorar ni minimizar las problemáticas que han acompañado el fenómeno - incluyendo la comisión de delitos graves - que se han originado a partir de dinámicas internas y de la difícil relación de la tribu con el contexto social, cultural y político, al que no es ajena la presión policial y mediática. Entre 2002 y 2003, a medida que la tribu iba nutriéndose de nuevos miembros, asentaba su estructura y su red organizativa y se distribuía en diferentes distritos de Madrid, además, iba encontrando en ciertas formas de violencia una respuesta eficaz al déficit individual y grupal de inserción social y a la discriminación. Dicho recurso comenzó a ser empleado también por la Nación, tanto intragrupalmente como intergrupalmente, en el contexto de los grupos compuestos por inmigrantes latinoamericanos, donde la comparación no resultara desventajosa en otras dimensiones que no fueran la fuerza física (como podría suceder con jóvenes autóctonos): «piques» y «vacile» constituirían el marco común de las así llamadas «bandas latinas». Los problemas de enfrentamientos con otros grupos, fundamentalmente compuestos por latinoamericanos, coinciden, más o menos, tanto con su mayor expansión como con el comienzo de la intervención policial: la Nación en Madrid llegó a tener unos veinte capítulos, distribuidos sobre todo en sus distritos del sur, y a tener entre sus filas entre 300 y 600 integrantes, de los cuales más o menos unos 100 eran reyes y reinas. En la actualidad, las dimensiones de la Nación se han reducido aproximadamente a una cuarta parte, debido, en un primer momento, al abandono casi masivo de la tribu por parte de sus miembros a causa de los conflictos internos y externos; en un segundo momento, a la resonancia social que comenzaban a adquirir tales conflictos, $y$, en un tercer momento, que se solapa en parte al anterior, a las actuaciones policiales. En las fases, podríamos decir, de apogeo de la tribu, se empezaron a dar con más frecuencia, constancia y extensión dinámicas internas propiciadas por la estructura jerárquica que primaron, en primer lugar, el respeto adscrito sobre el adquirido y lo autoritario sobre lo carismático, y, en segundo lugar, la supremacía física y la dinámica del conflicto como criterios tanto de adquisición del estatus como de asignación y control del poder. Ello, a su vez, propició que los nuevos miembros ingresaran en la Tribu bien por la protección que dispensaba, bien por la fama y la proyección pública que iba conquistando entre los demás grupos de latinoamericanos. A este respecto, algunos entrevistados señalan que, en las reuniones de los capítulos o las Universales, fueron perdiendo importancia las actividades relacionadas con el apoyo y la ayuda mutua, la iniciación en la «literatura» y la formación personal, ganando preponderancia las orientadas a los conflictos internos y con otros grupos. Una vez más, si nos atene- 
mos a las condenas, aunque siempre con mucha cautela e intentando aislar casos extremos, remiten a este conjunto de conductas delictivas y no a otras relacionadas propiamente con grupos delictivos o criminales. En efecto, todavía no constan condenas por hechos relacionados con tráfico de drogas.

Debemos concluir recordando que el manifiesto señala también que los reyes y las reinas de la recién fundada tribu se han unido para luchar contra el racismo, la desigualdad social y la hipocresía. Su propósito es ser reyes y reinas de provecho estudiando o aprendiendo un oficio para obtener trabajos dignos y formar una corporación a través de la cual poder ofrecer trabajo a los demás miembros y evitar que sufran humillaciones. Éstos siempre han estado entre los propósitos de la tribu y de los miembros que realmente asumían y defendían los principios propios de la Nación y que han luchado, incluso en los momentos más difíciles, para que pudieran cumplirse. Y éstos siguen siendo los propósitos de la actual Nación en Madrid. Por nuestro trabajo presente, podemos afirmar, sin lugar a dudas, que cuando se les permite a sus miembros buscar, encontrar y emplear los medios para lograrlos, se les apoya en revertir algunos de los procesos y de las dinámicas perversos señalados anteriormente, se les abre - en definitiva - una vía para la integración positiva, la respuesta es sorprendentemente efectiva y provechosa, es decir, cuando puede desplegar todas sus potencialidades, la Nación se convierte en un grupo propiamente educativo (véase Scandroglio y López, 2010). Ciertamente, el proceso actual no está exento de conflictos, ni en las relaciones internas, ni en las relaciones con otras tribus, ni en las relaciones con otros grupos, la sociedad y los actores sociales. En pocos meses, ha tenido que enfrentarse a situaciones conflictivas que habrían podido hacerlo peligrar seriamente. Sin embargo, en todas las ocasiones o en aquellas a las que hizo frente el grupo hasta el momento, la vía elegida o experimentada ha sido la del refuerzo, la mediación y la integración.

\section{Reyes y reinas latinos en Barcelona}

Nosotros, los jóvenes latinos, queremos y necesitamos que valoricen nuestras culturas y nos ayuden a integrarnos a la sociedad, teniendo confianza y no tachando al joven latino como parte de una pandilla o banda delictiva.

(Queen Melody, ponencia presentada en el seminario "Jóvenes latinos: espacio público y cultura urbana», Barcelona, noviembre de 2005)

El 14 de septiembre de 2006, se presentó en un casal de jóvenes de Barcelona, ante una audiencia atónita, una nueva entidad juvenil recientemente inscrita en el registro de asociaciones de la Generalitat: la Organización Cultural de los Reyes y Reinas Latinos de Cataluña. El acto no hubiera despertado demasiado interés si no fuera porque tal nombre evoca un imaginario social hasta hace pocos meses sinónimo de algo casi diabólico: la peligrosa banda de los Latin Kings. A la cita acudieron casi un centenar de periodistas de 
todos los medios de comunicación (incluyendo casi todas las televisiones y la corresponsal de la revista Time), que presenciaron atónitos la salida del armario de un puñado de reyes y reinas, con su presidenta Queen Melody al frente. Al acabar el acto, los responsables del Consell de la Joventut comentaban irónicamente que las actividades del resto de asociaciones juveniles de Barcelona nunca habían despertado tanto interés. ¿Qué había sucedido para que una peligrosa banda de criminales se hubiera convertido en una inofensiva asociación cultural? ¿Eran los mismos que en Estados Unidos integraban la banda más numerosa y temida y en Madrid un juez se proponía declarar «asociación ilícita»? ¿Qué había detrás de esta escenografía de amarillo y negro, collares y coronas? Como en Madrid, todo había empezado en la calle y en los tribunales. El 28 de octubre de 2003, fue asesinado en Barcelona el adolescente colombiano Ronny Tapias a la salida del instituto donde estudiaba, tras sufrir una agresión por parte de un grupo de jóvenes. Según la investigación policial posterior, el asesinato fue un acto de venganza de los miembros de una banda (los Netas), que, supuestamente, confundieron a Ronny con un miembro de otra banda (los Latin Kings) con el que se habían peleado días antes en una discoteca. El caso supuso el «descubrimiento mediático» del fenómeno de las «bandas latinas» y despertó una oleada de "pánico moral» que no ha cesado desde entonces. Al cabo de un mes, fueron detenidos nueve jóvenes de nacionalidad dominicana y ecuatoriana. Tres eran menores y fueron juzgados y condenados (entre ellos, el supuesto autor material del crimen). El juicio a los otros seis (mayores de edad), realizado en abril de 2005, se convirtió en un acontecimiento seguido con gran atención por parte de los medios de comunicación. Fue nuestra entrada espectacular en el mundo de los Latin Kings.

A continuación, transcribimos fragmentos de nuestro diario de campo:

La Audiencia de Barcelona, situada junto al parque de la Ciutadella que durante mucho tiempo albergó a las fuerzas de ocupación, es hoy un foco de atención. En la entrada, las cámaras de las televisiones recogen declaraciones e informan en directo. Frente al corredor de las escalinatas se arremolinan jóvenes latinoamericanos, estudiantes de derecho, periodistas y curiosos: hoy empieza el juicio por el caso Ronny Tapias. En una sala cerrada se está acabando de seleccionar el jurado popular que, como en todo juicio por asesinato, es el encargado de valorar las pruebas. A primera hora de la tarde empieza la sesión pública. La sala noble está repleta: los frescos que cubren el techo (con escenas de juicios del antiguo Israel, la Roma clásica y la época papal) dan un aire de solemnidad a un estrado singular. A la izquierda de la juez, nueve miembros del jurado (ocho mujeres, varias jóvenes), a los que espera una difícil papeleta; a su derecha, la fiscal, los abogados de la acusación particular (la familia Tapias) y popular (el Ayuntamiento de Barcelona); un poco más allá, los seis abogados defensores. Los seis acusados - tres muchachos dominicanos y tres ecuatorianos, de edades comprendidas entre los 20 y los 24 años- están sentados bajo sus abogados, inmóviles pero inquietos, algunos con traje y corbata y otros con su vestimenta habitual. Arriba, en una especie de anfiteatro, una docena de periodistas siguen la vista. La sala está llena: no queda ni una sola 
plaza libre y hay mucha gente de pie. Entre los asistentes, los padres de Ronny, acompañados por un chico. La mayoría de sillas están ocupadas por familiares y amigos de los acusados. Se respira un aire tenso y los rostros expresan la tensión acumulada. Tras los trámites habituales, la fiscal expone su alegato. Los hechos sucedieron en la tarde del martes 28 de octubre de 2003, a la salida del instituto donde estudiaba la víctima. El domingo anterior, en las inmediaciones de la discoteca Caribe Caliente del Hospitalet de Llobregat (ciudad que forma parte del Área Metropolitana de Barcelona), se produjo «un incidente entre un grupo de individuos pertenecientes a la banda de los "Latin King" al acusar estos a "Pavel" [un menor ya condenado] de pertenecer a la banda rival de ellos llamada de los Ñetas [...]. Los "Latin Kings" y los "Ñetas" son bandas callejeras juveniles, violentas y de origen sudamericano. Ambas bandas mantienen rivalidad entre sí que provoca, frecuentemente, enfrentamientos violentos con empleo de arma blanca. Existe una tercera banda, la de los "Rancutas", que es cercana a la de los "Netas". Tras este incidente, los acusados dominicanos "se concertaron para acudir al día siguiente a la salida de las clases del citado centro con la finalidad de desquitarse, poner respeto y vengarse de los referidos Latin King y agredirles. Los tres acusados y los dos menores (ya condenados) difundieron su plan entre las bandas de los Netas y de los Rancutas», y se citaron a la salida del instituto. Tras confundir a Ronny con un miembro de los Latin Kings que estudiaba en el centro, le persiguieron y le asestaron una puñalada que le produjo la muerte. (Alegato de la fiscal, cursiva nuestra)

La sesión de hoy está dedicada a los interrogatorios de los seis acusados. La fiscal sigue en cada interrogatorio los mismos parámetros: empieza preguntando el país de origen, el año de llegada en Cataluña, la situación legal y su ocupación durante el tiempo en que se desarrollaron los hechos. Las informaciones que los caracterizan son su edad (son jóvenes), su procedencia (son latinoamericanos), su condición (son inmigrantes), su situación (sin papeles y sin ocupación fija). Tanto la acusación como los abogados formulan muchas preguntas sobre la estética de los acusados, símbolos que los vincularían a las bandas. Véase por ejemplo el siguiente interrogatorio: «Pregunta de la defensa no 2: ¿Cómo iban vestidos los dos chicos del accidente del tren? Acusado $n^{\circ} 1$ : Normal y el otro iba de ancho. Pregunta de la defensa no 2: ¿Llevaba símbolos de bandas? Acusado no 1: Iba sólo de ancho». La acusación intenta demostrar que los acusados, antes del asesinado, seguían determinados patrones estéticos. Las defensas, en cambio, fundamentan sus argumentaciones sobre el hecho que sus clientes no eran miembros de bandas y no se dotaban de ninguno de estos elementos estéticos: el pañuelo o la gorra en la cabeza y «ir de ancho». De hecho, varios testimonios que reconocieron a los acusados en las rondas de identificación admiten haberlo hecho únicamente por la vestimenta que llevaban (que describen genéricamente como «hip-hop» o «rapera»). Cuando se acaba la audiencia, queda la sensación que ser miembros de una banda es de por sí un agravante (si no legal, al menos moral).

Hoy es la sesión de conclusiones del juicio. La fiscal se reafirma en su alegato pero matiza que lo importante no es si los acusados pertenecían o no a una banda, sino el actuar en grupo para cometer un asesinato, concertándose para organizar las tareas de vigilancia, apoyo y ejecución. Uno de los abogados defensores hace un alegato en el que se remonta a la época romana («Mejor 
que mueran diez inocentes a que se salve un culpable») y a la lucha contra la herejía cátara ("Quemadlos a todos: Dios distinguirá a los suyos») para rechazar el proceso colectivo a las bandas en el que, en su opinión, se ha convertido el juicio. Este mismo abogado cuestiona las pruebas en las que se sustenta dicha teoría: «¿Por qué el fiscal y la acusación mantienen la teoría de las bandas? Porque si no hubiera bandas no podría acusarse a todos [...] Son Ñetas, son una banda, hubo un asesinato. Por lo tanto condenémoslos [...] ¿Es posible condenar a individuos a 17 o 20 años de cárcel por una mera pertenencia a las bandas?». A las siete de la tarde recibimos un mensaje de móvil anunciando la lectura del veredicto. Tras tres días de encierro, el jurado popular ha llegado a una decisión unánime que parece salomónica. Consideran culpables a los tres acusados dominicanos (los «concertados» para atacar a Ronny) e inocentes a los tres ecuatorianos (los que, según la fiscalía, realizaron «funciones de vigilancia y apoyo»). Aparentemente, el veredicto no valora la cuestión de las bandas (ninguna de las 50 preguntas hacía referencia al tema y no se considera probado que los que fueron a la discoteca pidieran ayuda a miembros de los Netas y de los Rancutas). El padre de Ronny, siempre comedido, que ha seguido el juicio, considera la sentencia justa: «No todos los acusados han tenido el mismo grado de implicación en los hechos y no quiero que paguen justos por pecadores».

A raíz de este acontecimiento y de otros que se sucedieron después en Madrid y Barcelona, las alarmas del Ministerio del Interior y de los medios de comunicación, se fue creando una imagen estigmatizada de la juventud latinoamericana. Tras el fantasma de las bandas, una presencia ignorada: la de miles de muchachos y muchachas, llegados a Barcelona desde fines de los años noventa (gracias, fundamentalmente, a diversos procesos de reagrupación familiar), (des)terrados de sus lugares y redes sociales de origen en uno de los momentos más críticos de sus vidas (la siempre difícil transición a la vida adulta), y enfrentados en su lugar de destino a adultos (a)terrados (madres superocupadas, padres a menudo ausentes, profesores y asistentes sociales inseguros, vecinos con miedo) frente a su liminaridad jurídica e institucional. Tras esta presencia inquietante, un espectro: el de nuevas formas de sociabilidad que cruzan fronteras geográficas y temporales para reconstruir identidades globales que seguimos confundiendo con pandillas tradicionales. Según los datos oficiales de la policía de Barcelona, la cantidad de jóvenes que pertenecerían a «bandas latinas» oscila entre 400 y 1.000 (en su mayoría varones). Sin profundizar en que su organización también estaría conformada por miembros que no son latinoamericanos, representarían únicamente entre el $1 \%$ y el $2 \%$ de la población juvenil de estas nacionalidades (entre el $2 \%$ y el $4 \%$, si descartamos a los menores de quince años). Por lo cual sería pertinente peguntarnos y reflexionar sobre problemáticas emergentes en torno a este tema: ¿por qué el imaginario dominante construye el estereotipo del joven latinoamericano sobre el escaso $2 \%$ que supuestamente pertenece a las bandas e invisibiliza al $98 \%$ restante?, ¿en qué medida este imaginario no acaba provocando el efecto contrario al que persigue, es decir, fomentar la difusión del fenómeno entre jóvenes 
que antes no se habían visto atraídos por esta forma de vida, con lo cual se convierte en refugio para una identidad cuestionada, en una especie de profecía autocumplida? Esas fueron las preguntas que guiaron el estudio Jóvenes latinos en Barcelona: Espacio público y cultura urbana (Feixa et al., 2006).

En noviembre de 2005, con motivo de la presentación del estudio, tuvieron lugar varios actos trascendentes: unos clandestinos (una reunión de casi 200 miembros de los Latin Kings a la que por primera vez invitaban a investigadores y representantes de las instituciones; una reunión con un grupo más reducido de Netas a los que Luis Barrios llevaba un mensaje del liderato de Nueva York, y un seminario masivo en el que por primera vez miembros de los Kings y de los Netas dejaron de ser fantasmas y se convirtieron en presencias reales). Después del seminario, se inició un proceso complejo pero extraordinariamente interesante. Los Latin Kings empezaron a llevar a la práctica su proyecto de ser reconocidos como asociación: con el asesoramiento del Instituto de Derechos Humanos de Cataluña, redactaron unos estatutos que habían de ser fieles a sus principios y a las leyes catalanas. Durante varios meses, discutieron el borrador en reuniones de base (llamadas "capítulos») y en asambleas (llamadas «universales»). El debate coincidió en el tiempo con el debate en torno al estatuto catalán, lo que condujo a situaciones divertidas, por ejemplo, la denominación de la asociación: al principio, los componentes del grupo no entendían por qué no podían utilizar el nombre oficial (Todopoderosa Nación de los Reyes y Reinas Latinos). Mientras los asesores legales les aseguraban que el nombre no hacía la cosa, el argumento definitivo para ellos fue saber que tampoco Cataluña había sido reconocida como nación en el Estatut aprobado en Madrid. Un domingo de mayo de 2006, un cura comprometido hace tiempo con el colectivo emigrante y que ahora colabora con los reyes y reinas, después de asistir a la votación definitiva, nos envió un SMS que decía más o menos: «№ sé si tendremos estatut, pero ya tenemos estatutos!». Poco después, los estatutos se presentaron al registro de asociaciones, eran finalmente reconocidos por el departamento de justicia a fines de julio de 2006 y pronto saltaron a la prensa.

¿Qué implica este reconocimiento? ¿Puede una banda latina transformarse en organización cultural? Según los asesores jurídicos que han intervenido en el tema, más que de «legalización», debería hablarse de "constitución de asociación» (pues los Latin Kings no eran antes ilegales, sino, en todo caso, alegales, como la mayor parte de los grupos juveniles). Pero más importante que este reconocimiento jurídico es la dinámica social que el proceso ha liberado: unas identidades hasta ahora proscritas pasan a ser aceptadas; el estigma se convierte en emblema. Los muchachos y las muchachas que hasta ahora debían ocultar su pertenencia a los Latin Kings, pueden salir de la clandestinidad (lo que no deja de guardar cierta similitud con procesos anteriormente vividos por grupos políticos o religiosos prohibidos). El simple proceso ya ha tenido efectos positivos: los actos de violencia se han reducido y se han volcado a la creatividad cultural: campeonatos deportivos, representaciones teatrales, elaboración de un documental (La vida real), la grabación de un CD de hip-hop 
y reaggueton (presentado durante las fiestas de la Mercè de 2006), y sobre todo el proyecto de mediación intercultural a través de la música promovido por el Casal de Joves de Roquetes, en Nou Barris, territorio de viejas y nuevas migraciones y de viejos y nuevos movimientos juveniles, y apoyado por una discográfica alternativa, en el que participaron latin kingts, ñetas y otros jóvenes de Barcelona, y que tuvo como resultado la elaboración de un $\mathrm{CD}$, un documental y un libro, publicado con el título de Unidos por el Flow (VVAA, 2008, Cf. Feixa, 2009).

\section{Discusión: ¿Organización cultural o asociación ilícita?}

Son jóvenes organizados, en su estructura absolutamente piramidal, ferozmente jerarquizada y en el modelo terrorista de la organización.

(Jiménez Losantos, El Mundo, 7 de mayo de 2003)

¡Sólo les falta el pañuelo y son unos boy scouts!

(Educador de calle)

Los estudios de caso que hemos presentado ponen en evidencia dos polos extremos de los discursos y de las prácticas institucionales en torno al fenómeno de las denominadas «bandas latinas». Tal contraste no puede explicarse por diferencias entre los jóvenes y los grupos de los que forman parte de ellas - que responden a orígenes sociales y formas de organización similares-, sino que remite sobre todo a diferencias en el contexto sociopolítico, es decir, a las políticas de integración —o de estigmatización — impulsadas desde la sociedad de acogida. Sin embargo, aunque el resultante actual de los procesos ocurridos en Madrid y Barcelona aparezca como diametralmente opuesto, no debe considerarse como tal en su desarrollo, pues ambas tendencias - la legitimadora y la criminalizadora - conviven en ambos espacios urbanos en formas diversas.

Desde nuestro rol de investigadores y teóricos de la realidad social, creemos imprescindible explicitar los elementos conceptuales e ideológicos que subyacen a cada una de dichas propuestas y posicionarnos respecto a su eficacia a largo plazo en el afrontamiento de los problemas sociales. La postura criminalizadora, arraigada en concepciones clásicas del conflicto de tinte estructural y funcional (Touzard, 1980), está además influida por más recientes y muy poderosas ideologías transnacionales basadas en un nuevo «sentido común penal» —que Wacquant describe como una «operación planetaria de marketing ideológico» (2000: 14) —, y se caracteriza por la desatención por el análisis de los procesos inherentes a la propia estructura social que favorecen la aparición de los conflictos y el uso de una retórica militarizada de trazo grueso para la supuesta reconquista del espacio público para la gente «de bien», que apenas distingue entre pobres, marginales, delincuentes o inmigrantes. Promueve, en consecuencia, una representación estigmatizadora de los colectivos implicados 
en los mismos y explica su alejamiento de las prácticas sociales mayoritarias mediante elementos de irracionalidad y desviación, adscritos habitualmente a un nivel individual y teñidos con un carácter de irreversibilidad. Desde dicho planteamiento, se entienden como legítimas y eficaces las estrategias destinadas a la "eliminación", en términos sociales, de dichos colectivos, ya que su existencia en modo alguno es responsabilidad del orden social, ni afecta más que en forma negativa a su funcionamiento. Frente a este planteamiento, otras perspectivas desvelan que la estigmatización de determinados colectivos es una práctica que cumple determinadas funciones, inherentes a la propia dinámica social y a la interacción entre los diferentes colectivos sociales, y que sirve a efectos de afirmación identitaria, cohesión y legitimación del orden social mayoritario (Coser, 1961); la alarma social generada periódicamente en nuestro contexto hacia determinados fenómenos juveniles puede encuadrarse dentro de las críticas expiatorias dirigidas habitualmente en los momentos de transición económica y cultural hacia los sectores más vulnerables de la población (Walgrave y Mehlbye, 1998). Desde esta lectura, el abordaje de los problemas sociales debe iniciarse desde el análisis de las circunstancias que los generan y continuar con el diseño de estrategias de acción, proactivas y no reactivas, preventivas y no represivas, destinadas a transformar sus condicionantes, no meramente sus manifestaciones. La «eliminación» o «erradicación» de los colectivos considerados responsables del conflicto resulta así un absurdo, pues su existencia deriva de las condiciones generadas desde el propio sistema; sus manifestaciones tienen, a su vez, un carácter de reversibilidad, pues son producto de la interacción entre procesos que atañen a diferentes niveles y no exclusivamente al nivel individual. En consecuencia, la gestión de los problemas sociales debe plantearse desde el conocimiento de las oportunidades y expectativas implicadas en su núcleo y a través de prácticas que favorezcan el reconocimiento y la recuperación de poder (empowerment) de los colectivos implicados (Schnitman, 2000).

Situándonos en los contextos concretos que hemos examinado en este trabajo, debemos señalar que, si bien la actuación policial y judicial ha reducido la actividad de los Latin Kings en Madrid, no ha podido evitar ni que siguieran su misma estela otros grupos compuestos por inmigrantes latinoamericanos (en algunos casos, escenificando de forma más extrema y dramática el imaginario colectivo construido mediática y socialmente), ni que la Nación en Madrid desapareciera y, de paso, que volviera a aparecer la violencia de grupos juveniles de otro signo e integrados fundamentalmente por españoles. Si de prevenir el fenómeno y reducir la gravedad del mismo se trata, y no de otra cruzada, entonces la perspectiva adoptada en Barcelona ha sido no sólo más eficaz, sino menos costosa y más fructífera. El tiempo dirá si, además, es más sostenible. A pesar de que algunos profesionales de los cuerpos de seguridad o de instituciones públicas madrileñas, de lo cual somos testigos directos, opinen que podrían «acabar» con la Nación si se lo propusieran extendiendo su mano dura, nuestros conocimientos y nuestra experiencia, cuando no la historia misma de la humanidad, nos llevan a aventurar un futuro bien distinto para la 
Nación: cuando determinados referentes identitarios de carácter minoritario se convierten en "estigma» y crean una cohorte de «mártires», no sólo no se ven anulados o desacreditados socialmente, sino que pueden adquirir una mayor validez social.

Es por ello que defendemos, en primer lugar, un diagnóstico del fenómeno que lo encuadre bien dentro de los fenómenos juveniles propiamente culturales o bien dentro de la cultura de la violencia o del honor, colocando en primer plano los verdaderos factores subyacentes e incitando a la sociedad española a asumir sus responsabilidades como cuna de su cultivo y manifestación. En segundo lugar, proponemos unas líneas de prevención e intervención que persigan la acción proactiva destinada a potenciar los recursos y las capacidades de sus protagonistas, fortalecer sus redes grupales y comunitarias y ofrecer el espacio para que puedan convertirse en ciudadanos partícipes de su propio desarrollo. Esta perspectiva se encuadra dentro de una línea de acción que, ante el fracaso sostenido de numerosas iniciativas de reducción de la problemática juvenil, apuesta por la potenciación de los elementos positivos presentes en los entornos de socialización primarios, especialmente el grupo de pares, esenciales en la construcción normativa e identitaria de los jóvenes. Solamente una perspectiva integradora que atiende a los procesos psicosociales que subyacen a los conflictos evita que la exclusión y deslegitimización de colectivos y problemas los agrave y los cronifique.

\section{Postscriptum (1 de diciembre de 2010)}

En marzo de 2009 el Tribunal Supremo anuló el juicio a los Latin Kings de Madrid analizado en este artículo, por no probar de manera fehaciente que el objetivo fundamental del grupo fuese la comisión de delitos: «tal acerbo probatorio no justificaría por si sólo la calificación de la asociación ilícita sin quiebra de la garantía constitucional de presunción de inocencia», pues «no se hace la más mínima indicación que autorice a trasladarlos [los hechos delictivos] de su esfera estrictamente individual a la de la organización» (Sentencia $378 / 2009$, p. 20). Se trata de argumentos que en parte coinciden con los de nuestro informe pericial. La sentencia del Supremo obligaba a repetir el proceso, manteniendo únicamente para los procesados la imputación de asociación ilícita ya que, de las imputaciones de coacciones y amenazas, habían sido todos absueltos en la primera celebración del juicio. Mientras la sentencia condenatoria de 2007 recibió un tratamiento mediático relevante, su anulación por parte del TS apenas fue noticia. Además, en la segunda celebración del juicio, los medios, informados por el Ministerio Fiscal de la apertura de la vista, difundieron información que - en cuanto a imputados, imputaciones y petición de pena- se refería al primer juicio y no al segundo (ya no eran 14 los imputados, sino 10; ya no se imputaban, como señalamos, los delitos de coacciones y amenazas, sino solamente de asociación ilícita; ya no eran 9 el máximo de años solicitados por el Ministerio Fiscal, sino 4). Por otra parte, y en este mismo periodo, la experiencia de Barcelona se extendió a otros lugares: los Latin Kings 
\& Queens fueron reconocidos como asociación legalmente constituída en la Comunidad Valenciana y las Islas Baleares, y están en trámites de serlo en Murcia y Navarra. La repetición del juicio anulado por el Supremo ha empezado en octubre de 2010, con la participación como peritos de tres de los autores de este texto que, a diferencia del juicio anterior, hemos tenido ocasión de explicarnos con mayor profundidad. A día de hoy, el juicio está visto para sentencia.

\section{Referencias bibliográficas}

Álvarez, M.J. (2006). «Polémica por el intento de conducir a los "Latin Kings" hacia el asociacionismo. Morgades busca su integración y la Comunidad dice que no legaliza bandas». $A B C .4$ Octubre 2006. p. 40. En: http://hemeroteca.abc.es/ nav/Navigate.exe/hemeroteca/madrid/abc/2006/10/04/040.html. Accedido el 10 de febrero de 2010.

Brotherton, D.C. y Barrios, L. (2003). The Almitghty Latin King and Queen Nation: Street politics and the transformation of a New York City gang. Nueva York: Columbia University Press.

Cerbino, M. (2004). Pandillas juveniles: Cultura y conflicto de la calle. Quito: El Conejo \& Abya-Yala.

Coser, L. (1961). Las funciones del conflicto social. México: Fondo de Cultura Económica.

Cowie, H. y Wallace, P. (eds.) (2000). Peer support in action: From bystanding to standing by. Londres: Sage Publications.

DIFONZO, N. y BORDIA, P. (2007). Rumor psychology: Social and organizational approaches. Washington, DC: American Psychological Association.

FeIXA, C. (dir.); PorZIO, L. y ReCIO, C. (coords.) (2006). Jóvenes latinos en Barcelona: Espacio público y cultura urbana. Barcelona: Anthropos / Ajuntament de Barcelona.

FeIXA, C. (2006). De jóvenes, bandas y tribus: Antropología de la juventud. Barcelona: Ariel, 1998.

FeIXA, C. (2009). "Vida Real: reyes y reinas latinos en Cataluña (y más allá", Documentación Social. Madrid, 155: 227-260.

Ferrándiz, F. y FeIXA, C. (eds) (2005). Jóvenes sin tregua. Barcelona: Anthropos.

FISHER, R.J. (1991). The Social Psychology of Intergroup and International Conflict Resolution. Nueva York: Springer-Verlag.

Greene, J. y Pranis, K. (2007). Gang Wars. A Justice Policy Institute Report. [http://www.justicepolicy.org/content.php?hmID=1811\&smID=1581\&ssmID=22]

HagedORN, J.M. (2001). «Globalization, Gangs, and Collaborative Research». En: KLeIn, M.W.; Kerner, H-J.; MaXson, C.L. y WeiteKAMP, E. (eds). The Eurogang Paradox. Street Gangs and Youth Groups in the U.S. and Europa. Londres: Kluwer Academic Publishers, 41-58.

KueIN, M. (1995). The American Street Gang. Its Nature, Prevalence and Control. Nueva York: Oxford University Press.

Klein, M.W.; Kerner, H.-J.; MaXson, C.L.; Weitekamp, E. (eds.) (2001). The Eurogang Paradox: Street Gangs and Youth Groups in the U.S. and Europe. Londres: Kluwer Academic Publishers.

KonTOS, L. (2003). «Beween Criminal and Political Deviance: A Sociological analysis of the New York Chapter of the Almighty Latin King and Queen Nation». En: 
Muggleton, D. y Weinzierl, R. (eds.). The Post-Subcultures Reader. Londres: Berg, 133-150.

Madrid Diario/Europa Press (2007). «El Defensor del Menor pide que "no se haga la más mínima concesión policial" a las bandas latinas». Madridiario. 3 de mayo de 2007. En: http://www.madridiario.es/2007/Mayo/madrid/madrid/20261/el-defensor-del-menor-pide-que-no-se-haga-la-mas-minima-concesion-policial-a-las-bandas-latinas.html. Acccedido el 10 de febrero de 2010.

MATZA, D. (1973). «Subterranean traditions of youth». En: SilversteIN, H. (ed.). The Sociology of Youth: Evolution and Revolution. Nueva York: McMillan, 1961, 252-271.

QueIrolo, L. y TORRE, A.T. (eds.) (2005). Il fantasma delle bande. Giovani dall'America Latina a Genova. Génova: Fratelli Frilli Editore.

REguillo, R. (1995). En la calle otra vez. Las bandas: identidad urbana y usos de la comunicación. Guadalajara: ITESO, 1991.

Scandroglio, B.; López Martínez, J.S. y San José Sebastian, M.C. (2008). "Pandillas": grupos juveniles y conductas desviadas. La perspectiva psicosocial en el análisis y la intervención». Revista Electrónica de Investigación Psicoeducativa, 14, $6(1)$.

SCANDROGLIO, B; LÓPEZ, J. S. (2010). Investigación-acción-participativa con la agrupación Latin King en Madrid: potencialidades y límites de una estrategia alternativa al control de los grupos juveniles conflictivos. AIBR, Revista de Antropología Iberoamericana, 5(2):pp. 222-255.

SCHNitman, D.F. (2000). Nuevos paradigmas en la resolución de conflictos. Buenos Aires: Granica.

Thrasher, F.M. (1963). The Gang. A Study of 1313 gangs in Chicago. Chicago: University of Chicago Press, 1926.

TouzARD, H. (1980). La mediación y la solución de conflictos. Barcelona: Herder.

VIGIL, J.D. (1990). Barrio Gangs. Street Life and Identity in Southern California. Austin: University of Texas Press.

VV.AA. (2008). Unidos por el Flow: latin kings, ñetas y jóvenes de Barcelona, Barcelona, $\mathrm{K}$ Industria.

WACQUANT, L. (2000). Las cárceles de la miseria. Madrid: Alianza.

Walgrave, L. y Mehlbye, J. (1998). Confronting Youth in Europe: Juvenile Crime and Juvenile Justice. Copenhague: AKF.

WHYTE, W.F. (1972). La sociedad de las esquinas. México: Diáfora. Edición original: Street Corner Society. Chicago: University of Chicago Press, 1943. 